\title{
The Use of Electroencephalographic (Eeg) Wearable Technology in Stress Management of Nursing Students: A Mixed Methods Study
}

\author{
Michael Bermudez* \\ Assistant Professor, Department of Occupational Therapy, USA
}

*Corresponding author: Michael Bermudez, Assistant Professor, Department of Occupational Therapy USA.

Received Date: October 20, 2019

Published Date: October 30, 2019

\section{Introduction}

People may encounter stressful situations daily. However, some persistently occurring stressors can detrimentally affect an individual's quality of life. Lazarus RS \& Folkman S [1] mentioned that stress occurs when people are not able to effectively adapt to environmental demands that can potentially impede their general well-being. For this study, nursing students were chosen as participants, as they can be perceived as individuals who encounter a significant amount of stress during their academic endeavors. According to Sawatzky JV [2], stress is a psychological factor that can have negative effects on nursing students' academic performance and personal welfare.

This mixed methods study was conducted to determine if electroencephalographic (EEG) wearable technology, which can assist in detecting the users' relaxed, neutral, and active brainwaves, could be used as a stress management strategy by students in an accelerated Bachelor of Nursing program. The explanatory sequential mixed methods design, which involves a two-phase collection of data, was employed for this study. Quantitative data were initially obtained and analyzed using descriptive and inferential statistics. Then, the qualitative data were collected from the participants and analyzed using descriptive and longitudinal coding.

Nursing students in a classroom were given the following questionnaires to complete: the Perceived Stress Scale (PSS), an assessment used to measure people's degree of perceived stress at a moment in time Cohen S, et al. [3]; and an initial questionnaire (formulated by the researcher) through which students' rated their perceptions on wearable technology use and stress management.
Ten subjects for the quantitative phase of the mixed methods study were randomly selected from an initial 12 volunteers. The participants were then instructed to wear the EEG device (Muse ${ }^{\mathrm{TM}}$ headband) in their nursing environments three times a week for 6 weeks with each session lasting 5 minutes.

The researcher employed the following procedure for obtaining the qualitative data:

- The researcher conducted the initial interview.

- Weeks 1 to 6: The participants answered qualitative surveys about their perceived stress before and after each use of the device.

- Weeks 2 to 5: The participants answered the interim questionnaires.

- Week 4: The researcher conducted interim interviews.

- $\quad$ The participants answered the summative questionnaire and submitted their journals.

- In addition, the researcher conducted the summative interviews.

After collecting the initial, interim, and summative questionnaires, the researcher analyzed the data obtained from the Likert-scale questions and presented the information through descriptive statistics. The researcher then used descriptive and longitudinal coding (measurement of changes over time) to analyze the information from the questionnaires.

The following themes emerged after data analysis through coding: 
- $\quad$ Perceptions of Wearable Technology.

- Perceptions of Stress.

- $\quad$ Coping Mechanisms Used.

- Perceptions of Meditation.

- Perceptions of the Use of the EEG Device for Stress Management.

- $\quad$ Out of the 10 participants, seven participants submitted their journals and questionnaires regarding ratings of stress levels before and after using the EEG device and were each assigned a case study. Two of the case studies were categorized as incomplete as two participants did not participate in the interim and summative interviews.

Each case study corresponded to the participants' experiences of using the device over the span of six weeks. The information from each case study (questionnaires, interviews, and journal entries) was analyzed through descriptive and longitudinal manual coding. Changes in the perceptions of the efficacy of the device for stress management over the course of the 6-week study were also analyzed via longitudinal coding.

Seven participants submitted their questionnaires regarding their stress ratings before and after using the EEG device. Using inferential statistics, the researcher concluded that there was no significant difference between the number of trials that were rated as a decrease in stress levels and trials that were rated as no change in stress levels. No increase in stress levels were recorded.

Out of the seven participants, four generally had positive opinions regarding the use of the device. Two of the four found the device beneficial overall and wanted to continue using the device after the study. One enjoyed using the device but was going to practice traditional meditation instead. Another one of the four liked using the device but did not indicate any intention of wanting to continue using the device. Three out of the seven participants generally disliked using the device. Each of the participants formulated their own personal meaning and perceptions of the efficacy of the device based on their personal experiences of the study.

One factor that may have affected the perception of the EEG device as an effective stress management strategy was the participants' repertoire of coping mechanisms. The researcher noticed a possible pattern that subjects who generally had more coping mechanisms or performed these activities on a more frequent basis were more receptive to learning about and using the
EEG device for stress management. The researcher also concluded that the continuously high stress levels encountered by the participants appeared to affect the receptivity in learning and using the EEG device and incorporating its use as part of their coping mechanisms. As the participants continued to experience high stress levels in the nursing program, some probably prioritized other coping skills that were more meaningful and important to them rather than using the EEG device for meditation.

Four of seven remaining participants who had complete and incomplete cases found the device to be an effective stress management technique, while three did not. Three out of five participants with complete case studies found the device effective in managing stress, while two out of five did not. Each of the participants formulated their own meaning and views of the efficacy of the device as a stress management strategy based on their personal experiences. Despite differences of the participants' opinions regarding the use of the device, most of them agreed that the device can be an effective introduction to meditation for novice meditators, those with low to moderate stress, and individuals who regularly use coping mechanisms to address stress.

The nursing students had varying views regarding the use of the EEG device for stress management. However, most of them reflected on their own perceptions and attitudes about stress and coping skills as a result of undergoing this study. Overall, the researcher recommends that nursing students use the EEG device for stress management, although personal opinions and preferences regarding coping skills should be taken into consideration in choosing an appropriate coping strategy as a stress management strategy. The researcher also suggests that stress levels and the use of coping mechanisms can affect the nursing students' use of the EEG device as a stress management strategy while undergoing academic coursework.

\section{Acknowledgment}

None.

\section{Conflict of Interest}

No conflict of interest

\section{References}

1. Lazarus RS, \& Folkman S (1984) Stress: appraisal and coping. Springer Publishing Company, USA.

2. Sawatzky JV (1998) Understanding nursing students' stress: A proposed framework. Nurse Educ Today 18(2): 108-115.

3. Cohen S, Kamarck T, Mermelstein R (1983) A global measure of perceived stress J Health Soc Behav 24(4): 386-396. 